\title{
A MECHANISM-BASED THERMO-VISCOELASTIC CONSTITUTIVE LAW FOR FIBER REINFORCED POLYMER MATRIX COMPOSITES
}

\author{
L. Di Gennaro ${ }^{1 *}$, F. Daghia ${ }^{1}$, M. Olive ${ }^{1}$, F. Jacquemin ${ }^{2}$ and D. Espinassou ${ }^{3}$ \\ ${ }^{1}$ Université Paris-Saclay, ENS Paris-Saclay, CNRS, LMT - Laboratoire de Mécanique et Technologie, \\ 4 avenue des Sciences, 91190, Gif-sur-Yvette, France. \\ 2 Institut de Recherche en Génie Civil et Mécanique (GeM) - UMR CNRS 6183, 58 Rue Michel Ange, \\ 44600 Saint Nazaire, France. \\ ${ }^{3}$ CETIM, Technocampus Composites Chemin du Chaffault, 44340 Bouguenais, France. \\ *livio.di_gennaro@ens-paris-saclay.fr
}

In the automotive sector, the increasing use of composite materials involved the use of modern and automated production techniques like the Laser Assisted Tape Placement (LATP) which assure high production rate but induce residual stresses and strains in the manufactured structures. In the present work, in order to take into account high temperature gradients caused by the manufacturing process, a three-dimensional thermo-viscoelastic constitutive model for the unidirectional tape is developed. The Cartan decomposition [1] of the stress and strain tensors is used under the hypothesis of transverse isotropy in order to link the contribution of the composite material to its constituents (fibers and matrix). This particular decomposition enables us to assume a fully elastic fibers' behavior and a temperature dependent visco-elastic behavior for the polymeric matrix. The presented constitutive model is implemented in an Abaqus UMAT which is used, following the work presented in [2], to assess residual stresses in the transverse direction induced by different initial cooling rates imposed on the surfaces of an infinite unconstrained unidirectional plate (Figure 1).

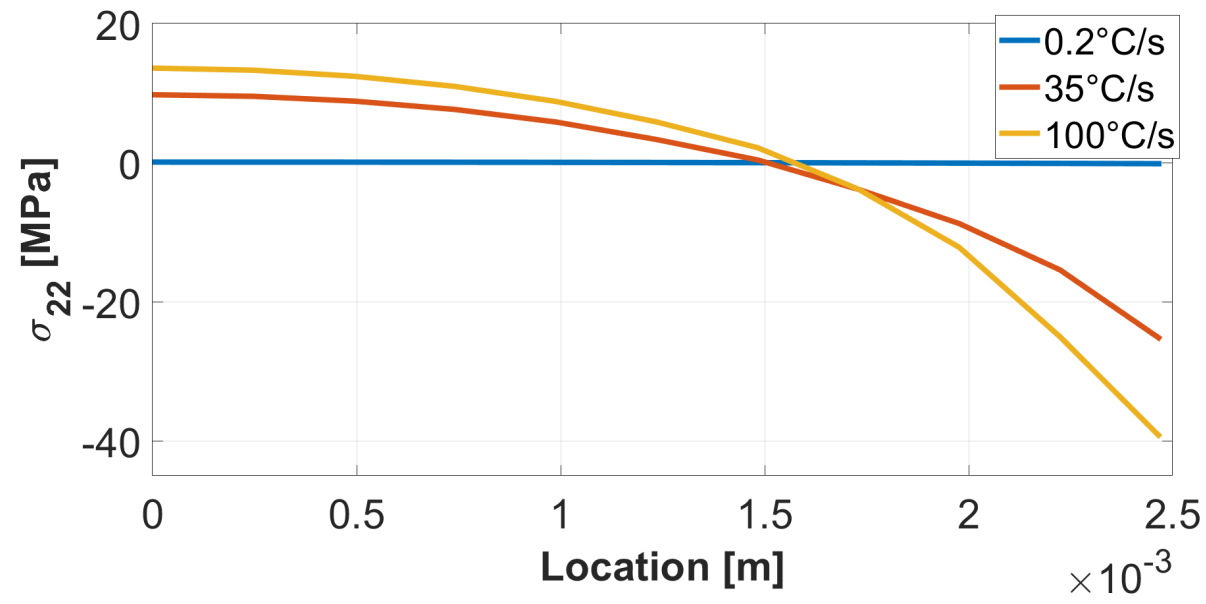

Figure 1: Stress through the thickness in the transverse direction for different initial surfaces cooling rates.

\section{References}

[1] M. Golubitsky, I. Stewart and D. G. Schaeffer, (1988) Singularities and groups in bifurcation theory. Vol. II. Springer-Verlag, New York.

[2] T. J. Chapman and J. W. Gillespie Jr. and R. B. Pipes and J.-A. Manson and J. C. Seferis (1990) Prediction of process-induced residual stresses in thermoplastic composites. Journal of Composite Materials, 24, 616-643. 